\section{UNIVERSITY OF} CALIFORNIA
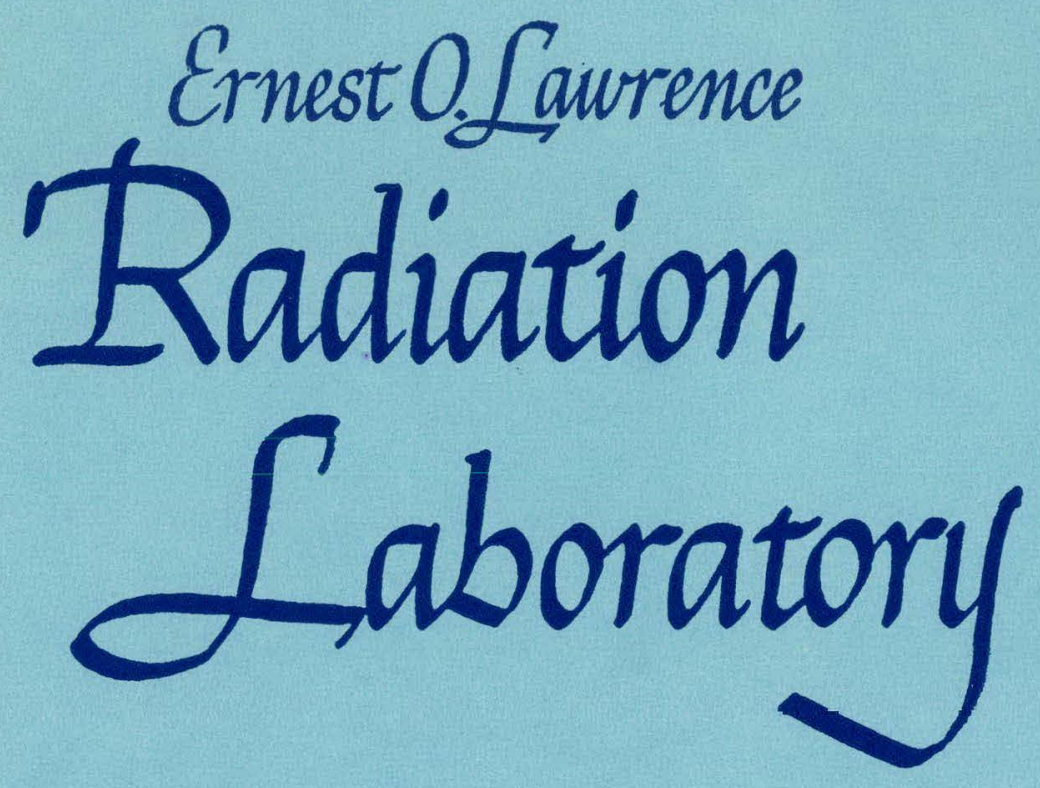

INVERSION OF THE

ANGULAR-MOMENTUM EXPANSION OF MESON PHOTOPRODUCTION AMPLITUDES

BERKELEY, CALIFORNIA 


\section{DISCLAIMER}

This report was prepared as an account of work sponsored by an agency of the United States Government. Neither the United States Government nor any agency Thereof, nor any of their employees, makes any warranty, express or implied, or assumes any legal liability or responsibility for the accuracy, completeness, or usefulness of any information, apparatus, product, or process disclosed, or represents that its use would not infringe privately owned rights. Reference herein to any specific commercial product, process, or service by trade name, trademark, manufacturer, or otherwise does not necessarily constitute or imply its endorsement, recommendation, or favoring by the United States Government or any agency thereof. The views and opinions of authors expressed herein do not necessarily state or reflect those of the United States Government or any agency thereof. 


\section{DISCLAIMER}

Portions of this document may be illegible in electronic image products. Images are produced from the best available original document. 


\author{
UNIVERSITY OF CALIFORNIA \\ Lawrence Radiation Laboratory \\ Berkeley, California \\ Contract No. W-7405-eng-48
}

\title{
INVERSION OF THE ANGULAR-MOMENTUM EXPANSION OF MESON PHOTOPRODUCTION AMPLITUDES
}

James S. Ball

August 18, 1959 

Printed in USA. Price 50 cents. Available from the office of Technical Services U. S. Department of Commerce Washington 25 , D.C.




\title{
INVERSION OF THE ANGULAR - MOMENTUM EXPANSION OF MESON PHOTOPRODUCTION AMPLITUDES
}

\author{
James S。Ball \\ Lawrence Radiation Laboratory \\ University of California \\ Berkeley, California
}

August 18, 1959

\section{ABSTRACT}

Helicity amplitudes are written for photoproduction of mesons and related to the amplitudes of Chew, Goldberger, Low, and Nambu. The expansions of both types of amplitudes in terms of amplitudes for particular angularmomentum states are then inverted. 


\title{
INVERSION OF THE ANGULAR-MOMENT UM EXPANSION OF MESON PHOTOPRODUCTION AMPLITUDES *
}

\author{
James S. Ball \\ Lawrence Radiation Laboratory \\ University of California \\ Berkeley, California
}

August 18, 1959

\section{INTRODUCTION}

The formulation by Jacob and $\mathrm{Wick}^{1}$ (hereafter referred to as JW) of scattering theory. in terms of helicity states results in a simplification of the amplitudes for photoproduction. The advantages gained by. employing these amplitudes are: (a) One is able to write directly a rather simple angularmomentum expansion for the various amplitudes; and (b). the complete set of functions [d. $\mathrm{J}(\theta)$ ] used in this expansion have simple or thogonality relations that make the inversion of these expansions quite simple. In this note, we explore the relationship between helicity amplitudes and the amplitudes heretofore used in photomeson theory.

\section{HELICITY AMPLITUDES}

By, the use of the procedure and notation of $\mathrm{JW}$, the four helicity states for the photon-nucleon system are described completely. by the total helicity (ג) of the system:

$$
\lambda=\lambda_{p}-\lambda_{\gamma}, \quad \lambda_{p}= \pm \frac{1}{2}, \quad \lambda_{\gamma}= \pm 1 .
$$

The nucleon is taken, for convenience, to be traveling in the $z$ direction so that $\lambda_{p}$ will be identical to the. $z$ component of nucleon spin. Since the $\pi$ has no spin, the total final helicity $(\mu)$ is just the helicity of the final nucleon.

If we assume parity conservation, the helicity, amplitudes. $\left(f_{\mu, \lambda}\right)$ obey.

$$
f_{-\mu,-\lambda}(\theta, \phi)=\eta_{g} \stackrel{f}{\mu, \lambda}_{\lambda}(\theta, \pi-\phi),
$$

where

$$
\eta_{g}=\frac{\eta_{n} \eta_{\gamma}}{\eta_{n} \cdot \eta_{\pi}}(-1)^{\mu-\lambda}
$$

If we make the usual intrinsic parity; assignment $\eta_{n}=1 \ldots$ and $\eta_{\gamma}=\eta_{\pi}=-1$, the scattering amplitude is

\footnotetext{
This work done under the auspices of the U.S. Atomic Energy Commission.
} 


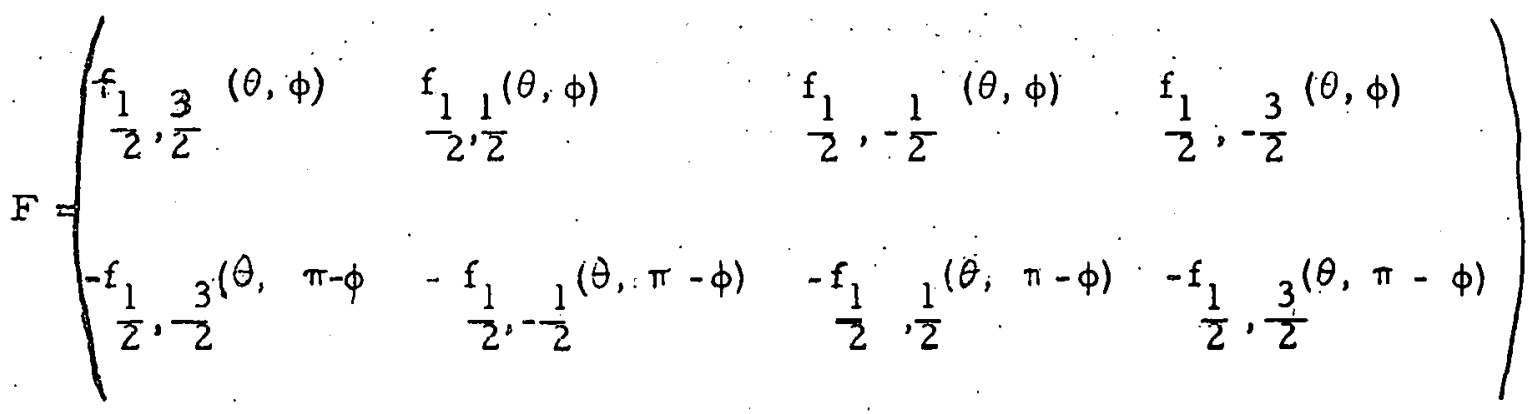

where

$$
\begin{aligned}
& \left.f_{\frac{1}{2}, \lambda}-\sum_{J}^{\Sigma(J}+\frac{1}{2}\right) \Lambda_{\lambda}^{J} \theta^{i\left(\lambda-\frac{1}{2}\right) \phi}{ }_{\lambda, \frac{1}{2}}(\theta) \\
& \text { Now, using the orthogonality relation for } d_{\lambda, \mu} J \\
& \int_{0}^{\pi} d_{m \mu}^{J}(\theta) d_{m \mu} J^{\prime}(\theta) \sin \theta d \theta=\frac{\delta_{J} J J}{\left(J+\frac{1}{2}\right)} .
\end{aligned}
$$

one gets

$$
A_{\lambda}^{J}=\frac{1}{2 \pi} \int_{0}^{\pi} \sin \theta d \theta \int_{0}^{2 \pi} d \phi d_{\lambda^{\prime}} \frac{1}{2}(\theta) e^{-i(\lambda-1 / 2) \phi} \frac{f}{2}, \lambda_{\frac{1}{2}}(\theta, \phi) .
$$

The differential cross section is given by

$$
\frac{\mathrm{d} \sigma}{\mathrm{d} \Omega}=\frac{\mathrm{q}}{\mathrm{k}}\left|\mathrm{f}_{\mu, \lambda}\right|^{2} .
$$




\section{CONNECTION BETWEEN AMPLITUDES}

The amplitudes of Chew, Goldberger, Low, and Nambu ${ }^{2}$ (hereafter referred to as CGLN), are defined by

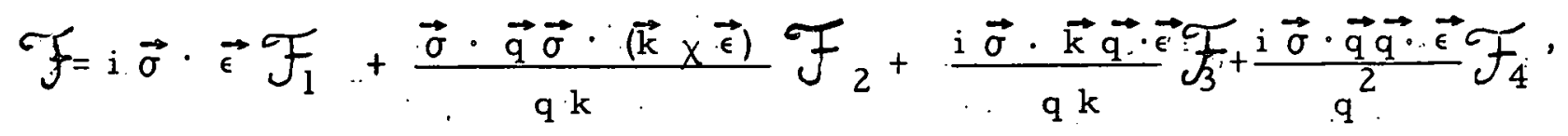

where the differential cross section is then defined by

$$
\frac{\mathrm{d} \sigma}{\mathrm{d} \Omega}=\frac{\mathrm{q}}{\mathrm{k}}\left|\mathrm{x}_{\mathrm{f}} \mathcal{F}_{\mathrm{x}}\right|^{2}
$$

The $\chi$ 's are the usual Pauli spinors representing the $z$ component of nucleon spin.

The helicity amplitudes differ from those of 'CGLN in that (a) they: refer to circularly polarized photons, and (b) the final nucleon spin is quantized.along the direction of motion rather than the $z$-axis.

For a definite: helicity of the photon, we can take the linear combinations of the spin-up and.spin-down Pauli spinors that correspond to the helicity states. When these linear combinations are used as the final state, the resulting amplitudes are just the helicity amplitudes. The linear combinations required are obtained by rotating the $z$ axis to lie along the direction of motion. A particular photon helicity. F, can be represented as

$$
F_{\mu, \lambda_{p}}{ }^{\lambda} \gamma=\chi_{\mu} F^{\lambda} \cdot x_{\lambda_{p}}
$$

where the $X^{\prime}$ 's are the usual two-component Pauli spinors. Also we can write

$$
F_{\mu, \lambda_{p}}^{\lambda}{ }^{\lambda}=x_{\mu} R^{-1} \mathcal{F}^{\lambda} \gamma x_{\lambda_{p}}
$$

and thus we have

$$
F^{\lambda} \gamma=R^{-1} \mathcal{F}^{\lambda} \gamma
$$

If for convenience in comparison we take $\phi=0$, then we have

$$
\mathrm{R}^{-1}=\mathrm{e}^{\mathrm{i} \frac{\theta}{2} \sigma_{y} .}
$$

The $\lambda_{y}=-1$ state of a photon traveling in the negative $z$ direction is represented by 
$-6-$

UCRL- 8858

$$
\vec{\epsilon}=\frac{\vec{\epsilon}_{x}+i \vec{\epsilon} y}{\sqrt{2}}
$$

The amplitude of CGLN for this case becomes:

$$
\begin{aligned}
F^{-} & =\frac{i}{\sqrt{2}}\left\{\left(\mathcal{F}_{1}+\mathcal{F}_{2}\right)\left(\sigma_{x}+i \sigma_{y}\right)+\sin \theta\left(F_{3}-\mathcal{F}_{4}\right) \sigma_{z}\right. \\
& \left.-2 \cos \frac{\theta}{2} e^{-i \theta \frac{\sigma_{y}}{2}}\left[\tilde{f}_{2}^{2}\left(\sigma_{x}+i \sigma_{y}\right)-\sin \dot{\theta} \mathcal{F}_{4} \sigma_{z}\right]\right\} .
\end{aligned}
$$

Substituting this in Eq. (3.5), we obtain

$$
\left.R^{-1} F^{-}=\frac{i}{\sqrt{2}} \mid \begin{array}{c}
\cos \frac{\theta}{2} \sin \theta\left(\mathcal{F}_{3}+\mathcal{F}_{4}\right)-2\left(\mathcal{F}_{1}-\mathcal{F}_{2}\right) \cos \frac{\theta}{2}-\sin \theta \sin \frac{\theta}{2}\left(\mathcal{F}_{3}-\mathcal{F}_{4}\right) \\
-\sin \frac{\theta}{2} \sin \theta\left(\mathcal{F}_{3}-\mathcal{F}_{4}\right)-2\left(\mathcal{F}_{1}+\sigma_{2}\right) \sin \frac{\theta}{2}-\sin \theta \cos \frac{\theta}{2}\left(\mathcal{F}_{3}+\mathcal{F}_{4}\right)
\end{array}\right)
$$

$$
=F^{-}=\left(\begin{array}{cc}
f_{1}, \frac{3}{2} & \frac{f_{1}}{2}, \frac{1}{2} \\
-f_{1},-\frac{3}{2} & \frac{f_{1}}{2},-\frac{1}{2}
\end{array}\right) \ldots
$$


This yields

$$
\begin{aligned}
& \frac{i}{\sqrt{2}} f_{1}=-\frac{f_{1}, \frac{3}{2}+f_{1} \frac{1}{2}, \frac{.1}{2}}{4 \sin \frac{\theta}{2}}+\frac{f_{1} \cdot \frac{1}{2}+\frac{f_{1}}{2},-\frac{3}{2}}{4 \cos \frac{\theta}{2}},
\end{aligned}
$$

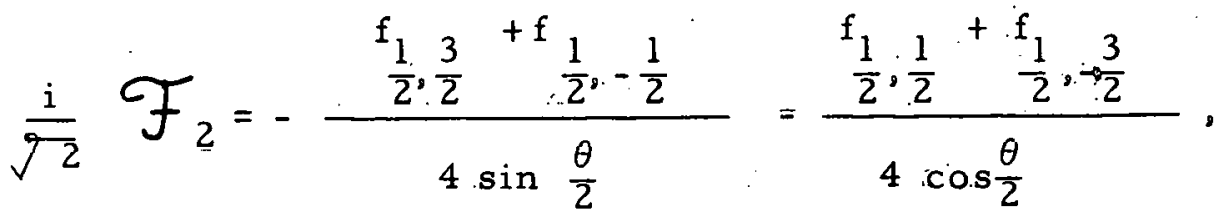

$$
\begin{aligned}
& \frac{i}{\sqrt{2}} F_{3}=\frac{1}{2 \cdot \sin \theta}\left\{\frac{f_{1} \cdot \frac{3}{2}}{\cos \frac{\theta}{2}}+\frac{f_{1} \cdot \frac{3}{2}}{\sin \frac{\theta}{2}}\right\} \text {, }
\end{aligned}
$$

and

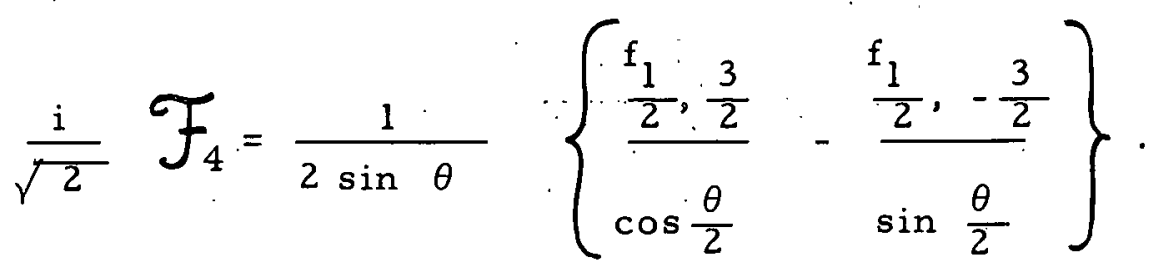

By expanding the $f^{\prime} s$, one obtains an expansion for the $\mathcal{F}^{\prime} s$ in terms of first and second derivatives of Legendre ploynomials which can then be compared to the expansion of the 'f's given by CGLN. This yields the relations:

$$
\left.\begin{array}{l}
M_{\ell+}=\frac{-\mathrm{i} / 2}{4(\ell+1)}\left\{\mathrm{A}_{\frac{1}{2}}^{\ell+1 / 2}-\mathrm{A}_{\frac{1}{2}}^{\ell+1 / 2}+\sqrt{\frac{l+2}{\ell}} \cdot\left(\mathrm{A}_{3}^{\ell+1 / 2}-\mathrm{A}_{3}^{\ell+1 / 2}\right)\right. \\
\frac{(3.11 \mathrm{a})}{2}
\end{array}\right\} .
$$




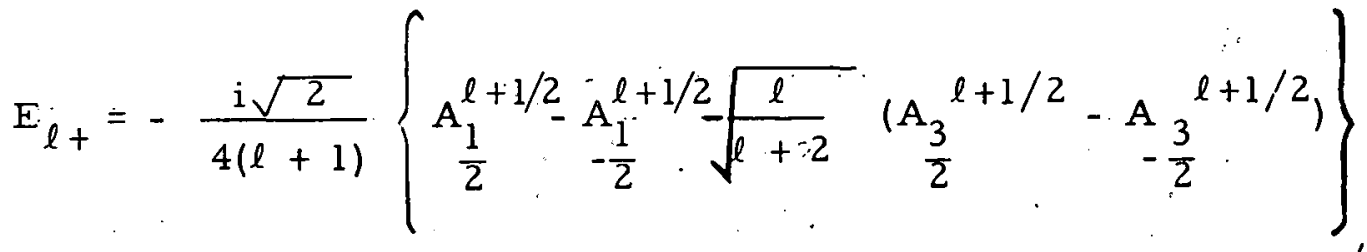

$$
\begin{aligned}
& E_{(\ell+1)-}=-\frac{i \sqrt{2}}{4(\ell+1)}\left\{A_{\frac{1}{2}}^{\ell+1 / 2}+A_{-\frac{1}{2}}^{\ell+1 / 2}+\sqrt{\frac{l+2}{\ell}} c_{-\frac{3}{2}}^{\ell+1 / 2}+A_{-\frac{3}{2}}^{\ell+1 / 2}\right\} ;
\end{aligned}
$$

where $\ell=J-\frac{1}{2}$.

\section{INVERSTON OF AMPLITUDES}

Equation (3.10) and Eq. (2.7) can be combined to express $A_{\lambda}{ }^{\ell}$ in terms of the $\mathcal{F}$ 's, yielding

$$
\begin{aligned}
& \mathrm{A}_{ \pm \frac{3}{2}}^{\ell+1 / 2}=-\frac{i}{\sqrt{2}} \sqrt{\ell(\ell+2)} \int_{1}^{1} \mathrm{dx} \frac{\left(\mathcal{F}_{3} \pm \mathcal{F}_{4}\right)}{2}\left\{\frac{p_{\ell}(x)-p_{\ell+2}(x)}{2 \ell+3}\right. \\
& \left. \pm \frac{\dot{p}_{\ell-1}(x)-p_{\ell+1}(x)}{2 \ell+1}\right\} \\
& \mathrm{A}_{ \pm \frac{1}{2}}^{\ell+1 / 2}=\frac{\mathrm{i}}{\sqrt{2}} \int_{-1}^{1} \mathrm{dx}\left\{\left(\mathcal{F}_{1} \mp \mathcal{F}_{2}\right)\left(+\frac{\mathrm{x} \mp 1}{2}\left(\mathcal{F}_{3} \mp \mathcal{F}_{4}\right)\right\}\left(\mathrm{p}_{\ell+1}(\mathrm{x}) \pm \mathrm{p}_{\ell}(\mathrm{x})\right)\right.
\end{aligned}
$$

and

Finally, these formulae can be combined with Eq. (3.11) to project the $E^{\prime} s$ and $M^{\prime} s$ out of the $F^{\prime} s$ :

$$
M_{\ell+}=\frac{1}{2(\ell+1)} \int_{-1}^{1} d x\left[\mathcal{F}_{1} p_{\ell}(x)-\mathcal{F}_{2} p_{\ell+1}(x)-\mathcal{F}_{3} \frac{p_{\ell-1}(x)-p_{\ell+1}(x)}{2 \ell+1}\right]
$$


-9-

UCR L $=8858$

$$
\begin{aligned}
E_{\ell+}=\frac{1}{2(\ell+1)} \int_{-1}^{1} \mathrm{dx}\left[\mathcal{F}_{1} \mathrm{p}_{\ell \ell}(\mathrm{x})-\mathcal{F}_{2} \mathrm{p}_{\ell+1}(\mathrm{x})+\mathcal{F}_{3} \cdot \frac{\left(\mathrm{p}_{\ell-1}(\mathrm{x})-\mathrm{p}_{\ell+1}(\mathrm{x})\right)}{2 \ell+1}\right. \\
\\
\left.+\mathcal{F}_{4}(\ell+1) \frac{\mathrm{p}_{\ell}(\mathrm{x})-\mathrm{p}_{\ell+2}(\mathrm{x})}{2 \ell+3}\right] .
\end{aligned}
$$

$$
M_{\ell-}=\frac{1}{2 \ell} \int_{-1}^{1} d x\left[-\mathcal{F}_{1} p_{\ell}(x)+\mathcal{F}_{2} p_{\ell-1}(x)+\mathcal{F}_{3} \frac{\left(p_{\ell-1}(x)-p_{\ell+1}(x)\right)}{2 \ell+1}\right]
$$

$$
\begin{aligned}
& E_{\ell-}=\frac{1}{2 \ell} \int_{-1}^{1} \mathrm{dx}\left[\mathcal{F}_{1} p_{\ell}(x)-\mathcal{F}_{2} \mathrm{p}_{\ell-1}(x)-\mathcal{F}_{3}(l+1) \frac{\left(\mathrm{p}_{\ell-1}(\mathrm{x})-\mathrm{p}_{\ell+1}(\mathrm{x})\right)}{2 \ell+1}\right. \\
& \left.-\because \mathcal{F}_{4} \frac{p_{l-2}(x)-p_{l}}{2 l-1}\right] \\
& \ell>\mathrm{I}
\end{aligned}
$$




\section{CONCLUSION!}

The above formulas give the recipe for projecting multipole amplitudes out of the CGLN amplitudes. Another: use for these expressions is in deriving partial-wave dispersion.relations. for photoproduction. This work is now in progress.

\section{REFERENCES}

1. J, Jacob and G. C. Wick, On the General. Theory of Collisions for rartıcles with Spin, Bruukliaveii National Laboratory preprint. BNL-4073, 1959.

2. Chew, Goldberger, Low, and Nambu, Relativistic Dispersiori-Relation Approach to Photomeson Production, Phys. Rev. 106, 1345 (1957). 
This report was prepared as an account of Government sponsored work. Neither the United States, nor the Commission, nor any person acting on behal $f$ of the Commission:

A. Makes any warranty or representation, expressed or implied, with respect to the accuracy, completeness, or usefulness of the information contained in this report, or that the use of any information, apparatuc, method, or process disclused in this report may not infringe privately owned rights; or

B. Assumes any liabilities with respect to the use of, or for damages resulting from the use of any information, apparatus, method, or process disclosed in this report.

As used in the above, "person acting on behalf of the Commission" includes any employee or contractor of the Commission, or employee of such contractor, to the extent that such employee or contractor of the Commission, or employee of such contractor prepares, disseminates, or provides access to, any information pursuant to his employment or contract with the Commission, or his employment with such contractor. 